\title{
Hypertension Research Awards for authors of outstanding papers in Hypertension Research
}

\author{
Kazuomi Kario Editor-in-Chief ${ }^{1}$
}

Received: 4 October 2021 / Accepted: 4 October 2021 / Published online: 17 October 2021

(c) The Japanese Society of Hypertension 2021

The winners of the Japanese Society of Hypertension (JSH) 12th Hypertension Research Awards were announced at the JSH general meeting, held on October 16, 2021, in Okinawa, Japan. These awards were established in 2010 to recognize significant contributions to the advancement of research in hypertension and related studies. Of the large number of articles published in Hypertension Research, official journal of JSH, from April 2020 (Vol. 43, No. 4) to March 2021 (Vol. 44, No. 3), the journal's editorial committee members selected following winners.

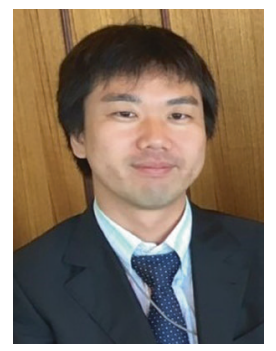

\section{Hypertension Research Excellence Award}

This was awarded to Dr. Norihiko Morisawa and colleagues from the Department of Pharmacology, Faculty of Medicine, Kagawa University, Kagawa, Japan for their paper titled "Renal sympathetic nerve activity regulates cardiovascular energy expenditure in rats fed high salt" (Hypertens Res. 2020;43:482-491). Their preclinical research showed that Sprague-Dawley rats fed a high salt diet showed a decrease in heart rate (HR) and a reduction in renal sympathetic nerve activity (RSNA) to curb cardiovascular energy expenditure, which attenuated the high salt-induced catabolic state and

Kazuomi Kario

kkario@jichi.ac.jp

${ }^{1}$ Hypertension Research https://www.nature.com/hr/ body weight loss. In contrast, high salt-fed Dahl salt-sensitive (DSS) rats did not show any reduction in HR and RSNA was increased. In these animals, renal denervation significantly decreased HR and attenuated the catabolic state independently of blood pressure, suggesting that the renal sympathetic nervous system plays an important role in the regulation of HR during high salt intake. These researchers concluded that renal denervation may benefit salt-sensitive hypertension by mitigating catabolism via suppression of cardiovascular energy expenditure.

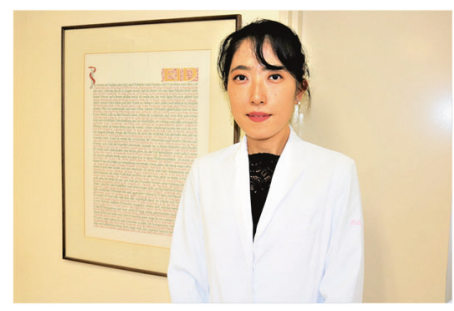

\section{Hypertension Research Award}

The 2021 recipient of this award was Dr. Hikari Takeshita and colleagues, from the Department of Geriatric and General Medicine, Osaka University Graduate School of Medicine, Suita, Osaka, Japan, for their paper titled "Different effects of the deletion of angiotensin-converting enzyme 2 and chronic activation of the renin-angiotensin system on muscle weakness in middle-aged mice" (Hypertens Res. 2020;43:296-304). This experimental study investigated the role of ACE2 in age-related loss of muscle mass and function (sarcopenia) using ACE2 knockout (KO) mice, who were compared with Tsukuba hypertensive $(\mathrm{TH})$ mice that overproduce human angiotensin II. In the gastrocnemius muscle, increased central nuclei (a marker of muscle degeneration) and expression of p16INK4a (a senescence-associated gene), along with alterations in the expression of several sarcopenia-associated genes, were observed in the ACE2KO mice but not in $\mathrm{TH}$ 
mice, indicating that chronic overactivation of the reninangiotensin system does not primarily contribute to the early aging phenotypes of skeletal muscle in ACE2KO mice.

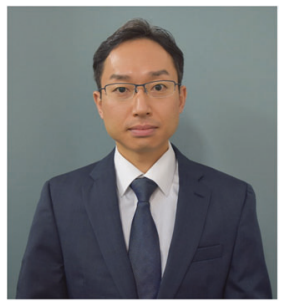

\section{Hypertension Research Award}

The final award for the last year was presented to Dr. Yasushi Matsuzawa and colleagues (Division of Cardiology, Yokohama City University Medical Center, Yokohama, Japan). Their paper was titled "Renin-angiotensin system inhibitors and the severity of coronavirus disease 2019 in
Kanagawa, Japan: a retrospective cohort study" (Hypertens Res. 2020;43:1257-1266). In Japan, the first coronavirus disease 2019 (COVID-19) outbreak occurred on the Diamond Princess Cruise Ship at Yokohama harbor in February 2020. Since the beginning of this outbreak, the authors have been working hard to treat patients with COVID-19 patients and investigated whether pretreatment with angiotensinconverting enzyme inhibitors (ACEi) or angiotensin receptor blockers (ARBs) reduced lung injury due to infection with SARS-CoV-2 and improved clinical outcomes in their patients $(n=151)$. They found that older age ( $>65$ years) was the most important risk factor for the development of severe COVID-19 disease on multivariate regression analysis (odds ratio 6.63 [95\% confidence interval 2.28-22.78]; $p<0.001$ ). In addition, prior use of ACEi/ARBs was associated with a significantly lower rate of new-onset or worsening mental confusion. This study shows the dedication to collecting realworld data and performing real-world clinical studies during the COVID-19 pandemic, and provides useful information to apply in managing future patients. 